\title{
THE SIGN OF CHARGE CARRIERS IN LUMINESCENT TRANSITIONS
}

\author{
S. NUDELMAN \\ Armour Research Foundation, Chicago 16, Illinois, U.S.A. \\ D. Brown, D. CURrIE \\ Institute of Science and Technology, The University of Michigan, Ann Arbor, \\ Michigan, U.S.A.
}

\begin{abstract}
A thorough treatment of the luminescent mechanisms in high resistivity semiconductors (up to $10^{12} \mathrm{ohm} \mathrm{cm}$ ), has as yet not been satisfactorily established. This is due to the lack of data as is provided by the Hall effect. An AC-AC Hall apparatus has been assembled to determine the sign of charge carriers, and their densities when involved in luminescent transitions. Results of preliminary measurements on CdS will be presented.
\end{abstract}

\section{INTRODUCTION}

This paper is concerned with the identification of the signs of charge carriers during luminescent transitions, and the determination of energy levels pertinent to these transitions in insulating and near insulating luminescent semiconducting materials. It is particularly concerned with obtaining this information by measurements of the Hall effect during excitation of luminescence and of correlating this Hall effect data with that obtained from the customary luminescence experiments.

Experimental studies on semiconductors such as germanium and silicon usually start with Hall effect measurements and add experiments to generate whatever other information is needed. Studies of near insulating materials, however, usually do not start with Hall data because of experimental difficulties caused by polarization and space charge effects, capacitive loading and electrical noise. High resistivity zinc sulfide and cadmium sulfide are examples of the latter semiconductors. Because of the unavailability of Hall data for these materials, the role of electrons and holes in the basic mechanisms responsible for their luminescent properties is of necessity often based upon imaginative interpretation of limited experimental information.

Fortunately, these experimental difficulties do not appear to be insurmountable. An AC-AC Hall apparatus that has been in use for three years is being optimized for usage with very high resistivity samples. It is expected that measurements will be possible on samples with an upper limit in resistivity in the order of $10^{11}-10^{12}$ $\mathrm{ohm}-\mathrm{cm}$. A description of the apparatus, some discussion of its principles of operation and preliminary data taken on a sample of $\mathrm{CdS}$ will be presented here.

\section{EXPERIMENTAL APPROACH}

a) Apparatus: A block diagram of the apparatus is shown in Fig. 1. It demonstrates a conventional arrangement for the placing of the Hall sample in the A.C. magnetic field and applying an A.C. electric field across the current electrodes. However 
a synchronous detector-low pass fitter system is incorporated in the measurement circuitry to obtain high noise voltage rejection by using long integration times. The system also contains a tuned circuit plus rectifier which is adequate for high signal to noise level measurements.

The magnet operates at $60 \mathrm{~Hz}$ and is capable of providing peak fields across a $3.5 \mathrm{~cm}$ air gap of up to 10,000 Gauss. It is a reproduction of a magnet designed by Levy [1] with pole faces of $5 \times 9.5 \mathrm{~cm}^{2}$ in cross-sectional area. A simple light

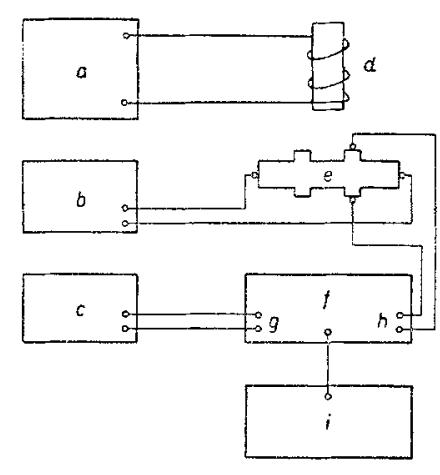

Fig. 1. Hall apparatus - schematic

a) $60 \mathrm{~Hz}$ magnet power supply

b) $40 \mathrm{~Hz}$ oscillator

c) $100 \mathrm{~Hz}$ oscillator

d) AC magnet

e) Hall sample

f) Synchronous detector

g) Sync input

h) Signal input

i) Display and recorder modulator-photocell system is used to generate a $40 \mathrm{~Hz}$ signal for the crystal current and a $100 \mathrm{~Hz}$ reference signal for the synchronous detector.

The Hall voltage consists of $20 \mathrm{~Hz}$ and $100 \mathrm{~Hz}$ components since it is generated from the product of two sine waves corresponding to the magnetic and electric fields [2]. The $100 \mathrm{~Hz}$ voltage is selected for measurement. The $100 \mathrm{~Hz}$ reference and Hall voltage signals are fed from tuned-amplifier circuits into the synchronous detector. The basic process in the synchronous detector is the formation of a product term as in the case of a heterodyne detector. In the synchronous detector, however, the two frequencies are equal and "locked in" in phase. The product of two sine (or cosine) waves gives rise to a D.C. term plus a double frequency term when the two sine waves are of the same frequency and phased properly. The output of the synchronous detector contains a low pass filter which filters out the high frequency term, and the Hall voltage is directly proportional to the Hall coefficient.

Typical luminescence measurements are usually made under constant temperature conditions, or for temperatures that rise linearly and slowly with time (as for glow curves) [3]. Thus the change in luminescence with temperature is essentially a very low frequency, or practically a D.C. measurement. The synchronous detector-low pass filter system will follow these changes with negligible error.

b) Principles of Operation: Electrical measurements on high resistivity materials are primarily limited by electrical noise [4]. The noise of most concern here is Johnson noise, since it is a direct function of resistance. The other types of noise are all dependent on current. In these high resistivity measurements, sample currents will be small to the extent that Johnson noise will be the predominant type of noise. This noise is described by the expression: $\bar{v}^{2}=4 k \operatorname{Tr} \Delta f$, where $v=$ $=v(i)-v_{\text {ave }}$ is the voltage fluctuation, $r=$ resistance of sample, $\Delta f=$ bandwidth measurement, $k=$ Boltzmann's constant, $T=$ temperature in absolute degrees.

The noise fluctuation is proportional to the bandwidth of the measurement. There 
is little that one can do to lower resistance values, other than to arrange the shape of the sample for minimum resistance. However, there are limits to this procedure, before significant error is introduced into the measurement [5]. Significant reduction in noise can be achieved by narrowing the bandwidth for the measurement. This is the primary purpese of the synchronous detector, and its low pass filter. This system has several unique advantages. The synchronous detector does not rectify noise, so that its response to a signal is linear. It can be made insensitive to signal frequency drift regardless of the system bandwidth. The bandwidth is determined by the low pass filter which may be conveniently narrowed with only the increasing response time of the system as a limit. The relationship between bandwidth and response time is approximated by: $2 . \Delta f . T=1$. Thus the procedure calls for narrowing bandwidth to the extent necessary for an accurate measurement, and correspondingly taking an increasing time to make the measurement. The system is capable of stable bandwidths as narrow as $0.003 \mathrm{~Hz}$, which leads to a minimum detectable Hall signal of $0.5 \mu \mathrm{V}$ for $10^{9} \mathrm{ohm}$ samples. Further reduction in noise can be achieved in the case of samples or electrodes that generate ap-

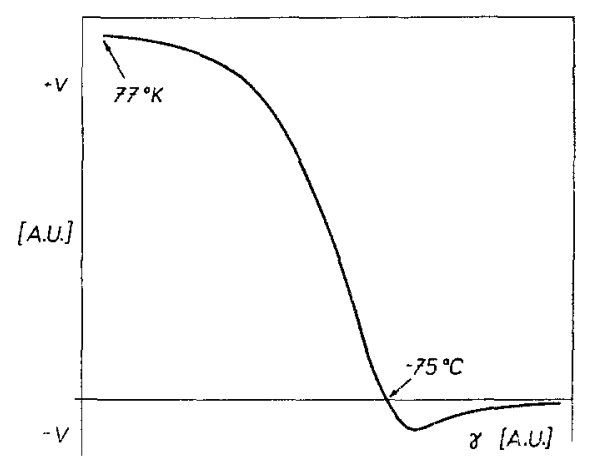

Figure 3. Example of Hall data presentation $+\mathrm{V}$ : positive Hall voltage $-\mathrm{V}$ : negative Hall voltage $\gamma:$ thermocouple EMF preciable $1 / f$ noise, by measuring the Hall voltage at as high a frequency as possible. For this reason, the sum frequency of $100 \mathrm{~Hz}$ is used, rather than the difference frequency of $20 \mathrm{~Hz}$.

It is well known that AC-AC Hall measurements minimize or effectively eliminate the errors introduced from thermoelectric or galvanometric effects $[2,5]$. However, additional advantages exist. In particular, it mitigates against the formation of polarization charges, and minimizes the general problems of space charge effects. Furthermore, the method eliminates potential drifts that are associated with D.C. measurements. Effective automation is inherent in the system in that the Hall voltage output from the synchronous detector system can be fed directly to X-Y recorders and plotted as a function of other kinds of data obtained simultaneously, such as temperature or intensity of luminescence.

A picture of the entire apparatus is shown in Fig. $2 .{ }^{1}$ ) A typical plot of Hall voltage versus temperature obtained from this apparatus is shown in Fig. 3. The Hall sample used was made with $40 \mathrm{ohm}-\mathrm{cm}$ resistivity tellurium, and shows a $p$ to $n$ transition. It was made by allowing the sample to warm up from liquid nitrogen to room temperature, by allowing the liquid nitrogen to evaporate from the dewar.

1) For Fig. 2 see Appendix III (p. 172b). 


\section{LUMINESCENCE}

There are three problems of interest that Hall data can help solve. The first is to determine the sign of the carrier or carriers involved in luminescent transitions involving the conduction or valence bands. This provides a firm basis from which the pattern of electronic transitions can be more fully established. The second problem is to help determine trap identity, density, and distribution. Measurements of thermoluminescence, photoluminescence, photoconductivity and electrical noise made simultaneously and automatically with that of the Hall effect will have more meaning since the contribution of holes and electrons to these phenomena can be more readily isolated. Third, it would be very helpful to monitor the density of charge carriers as a function of voltage in electroluminescent single crystal phosphors.

Measurements are in progress on a sample of CdS, with a resistivity of about $10^{5} \mathrm{ohm}-\mathrm{cm}$. A Hall voltage has been obtained at room temperature which is a function of photoexcitation. Tests are being made to determine the present capability of the system and the steps necessary for reaching theoretical limits in performance.

We would like to thank $\mathrm{J}$. Hickmott for his assistance in designing and assembling the synchronous detector filter system.

\section{Discussion}

Gergely: Have you had problems with non-ohmic electrodes?

Nudelman: Yes, problems of this nature exist. The method of solution has been to use a five electrode arrangement in which the current electrodes and Hall electrodes are ungrounded. Nitsche: How large are your Hall samples?

Nudelman: They vary from six to $10 \mathrm{~mm}$ long, sometimes longer and are several $\mathrm{mm}^{2}$ in cross section.

Received 12. 10. 1962.

\section{References}

[1] Levy J. L.: Phys. Rev. 92 (1953), 215.

[2] Lindberg O.: Proc. IRE 40 (1952), 1414.

[3] Haake C. H.: Enlarged Abst. of Electrochem. Soc., Spring Meeting (1957), p. 60.

[4] Nudelman S.: App. Opt. 1 (1962), 627.

[5] Putley E. H.: The Hall Effect and Related Phenomena, Butterworths, (London, 1960). 


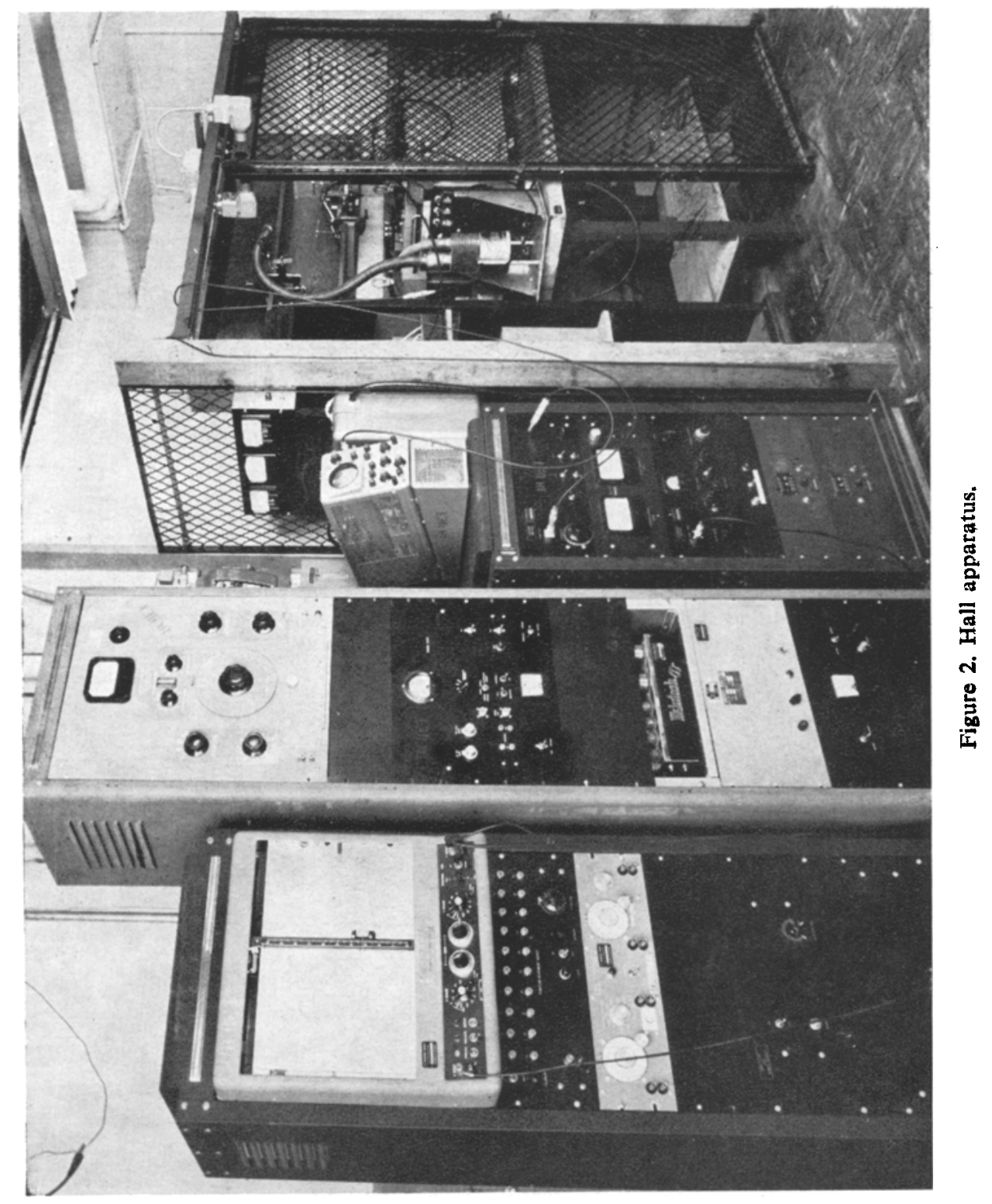

